\title{
PARTICIPACIÓN, VIDA DEMOCRÁTICA Y SENTIDO DE PERTENENCIA SEGÚN TIPO DE ESTABLECIMIENTO EDUCATIVO EN CHILE
}

\author{
Participation, Democratic Life and Sense of Belonging \\ according to type of educational institution in Chile
}

\author{
VICTOR CASTILLO RIQUELME*1 \\ CARLOS RODRÍGUEZ GARCÉS*2 \\ JUAN ESCALONA BURGOS $* * 3$ \\ ${ }^{*}$ CIDCIE - Universidad del Bío-Bío. Chile. \\ Correspondencia: vicastil@ubiobio.cl; carlosro@ubiobio.cl \\ **Universidad del Bío-Bío. Chile. \\ Correspondencia: juanescalona92@gmail.com
}

Recibido: 30-08-2016

Revisado: 18-11-2016

Aceptado: 19-12-2016

Resumen: Este artículo analiza, a través de bases de datos oficiales del Ministerio de Educación de Chile, la Participación y Formación Ciudadana como criterio de calidad educativa, comparando índices y estadísticas según establecimiento al que concurren los estudiantes de $8^{\circ}$ básico en Chile. Los hallazgos dan cuenta de diferencias estadísticamente significativas en las dimensiones de Vida Democrática, Sentido de Pertenencia y Participación. Si bien Participación y Formación Ciudadana constituyen uno de los objetivos fundamentales de la escuela, su manifestación no escapa a la dinámica de reproducción social instalada en el sistema educativo. Resulta preocupante que el fortalecimiento de la democracia y del comportamiento cívico de los estudiantes esté circunscrito al tipo de establecimiento que asisten.

Palabras clave: sistema educativo; educación ciudadana; democracia; organización de estudiantes; participación estudiantil.

\begin{abstract}
This article analyzes, through official databases of the Chilean Ministry of Education, Citizen Participation and Training as a criterion of educational quality, comparing indices and statistics according to the establishment to which the 8th grade students in Chile attend. The findings account for statistically significant differences in the dimensions of Democratic Living, Sense of Belonging and Participation. Although Participation and Citizenship Training are one of the fundamental objectives of the school, their manifestation does not escape the dynamics of social reproduction installed in the educational system. It is worrying that the strengthening of democracy and civic behaviour of students is limited to the type of school they attend.
\end{abstract}

Keywords: educational system; civic education; democracy; student organization; student participation.

\footnotetext{
${ }^{1}$ Licenciado en Educación, investigador adscrito al Centro de Investigación Educativa CIDCIE de la Universidad del Bío-Bío (Chile).

2 Director del Centro de Investigación CIDCIE de la Universidad del Bío-Bío (Chile).

${ }^{3}$ Licenciado en Educación, investigador adscrito al Centro de Investigación Educativa CIDCIE de la Universidad del Bío-Bío (Chile).
} 


\section{INTRODUCCIÓN*}

La escuela tiene dentro de sus finalidades fundamentales formar ciudadanos capaces de participar plenamente en sociedad y ejercer sus derechos y responsabilidades. Se encarga también de los propósitos axiológicos y éticos de la convivencia humana, los que a la luz de una buena formación deberían expresarse en valores civiles de respeto, tolerancia, pluralidad y buena convivencia. La escuela se constituye en un modelo a escala de la sociedad (Sahberg citado en Rosas y Santa Cruz, 2013), donde los estudiantes pueden aprender el sentido de la vida comunitaria y adquirir una membresía que los provea de derechos y deberes.

Chile, en su nivel de enseñanza básica o primaria, estructura su sistema educativo con base en la propiedad pública o privada y su financiamiento. Se distinguen así tres grandes conglomerados de establecimientos: municipales, particulares subvencionados y particulares pagos.

- Las escuelas municipales son establecimientos públicos y gratuitos, con alta presencia de alumnos vulnerables. De acuerdo con la tipificación de grupo socioeconómico (GSE) proporcionada por el Ministerio de Educación de Chile, el $87 \%$ de los establecimientos municipales son de tipo A (GSE-Bajo) o B (GSEMedio Bajo). En términos de concentración, el $72 \%$ de los establecimientos de GSE-Bajo (A) son escuelas municipales, es decir, establecimientos donde la mayor parte de los apoderados declaran tener menos de 8 años de escolaridad, disponer de un escaso ingreso familiar y vivir en condiciones de pobreza; y al menos el $79 \%$ de sus estudiantes presenta condición de vulnerabilidad social.

- Los colegios particulares subvencionados son de carácter privado y reciben financiamiento estatal mediante vouchers con base en la asistencia de alumnos. Estos establecimientos son más diversos en la configuración socioeconómica de su alumnado, en una gran proporción contemplan la participación familiar en el financiamiento mediante la modalidad de copago y concentran en la actualidad aproximadamente el $55 \%$ de la matrícula de enseñanza básica. De los 3.038 colegios particulares subvencionados existentes en Chile al año 2013, un 59\% pertenecían a la tipología C (GSE-Medio) o D (GSE-Medio Alto). La mayoría de los apoderados que pertenecen a estos establecimientos informan haber concluido la enseñanza media o tener estudios superiores y su estudiantado registra moderados niveles de vulnerabilidad social.

*Este artículo presenta parte de los resultados del proyecto de investigación "Aportes Socioeducativos a la integralidad de la educación: un análisis comparativo de la segmentación del desarrollo socio-personal" desarrollado por los autores durante el transcurso del año lectivo 2015 (marzo-diciembre) bajo el patrocinio del Centro de Investigación Educativa CIDCIE de la Universidad del Bío-Bío (Chile). 
- Los establecimientos particulares pagados concentran alrededor del 8\% de la matrícula, brindando sus servicios educativos a población de altos ingresos, siendo financiados en exclusividad por los aportes familiares del estudiante. El 90\% del GSE-Alto pertenece a esta tipología de establecimiento. La mayoría de los apoderados declaran tener educación superior completa, son profesionales de carácter universitario y menos del $11 \%$ de los estudiantes se encuentra en condición de vulnerabilidad social.

Esta estructuración administrativa imperante en Chile desde la reforma educativa implementada en los años 80 y prolongada por los gobiernos posdictadura, fundada en los principios de subsidiariedad y de libre elección, no ha tenido los logros educativos esperados. Además, se le consigna como responsable de profundizar los procesos de segmentación socioeducativa y de desigualdad de oportunidades (Hernández y Raczynski, 2015). Se trata de una segmentación socioeducativa con base en la capacidad de pago de las familias, que hace perder a la escuela su inherente capacidad de ser un espacio de convivencia donde confluyen los diferentes sectores sociales, aprenden a tolerarse, a resolver problemas y se generan lazos de amistad, sentimientos de identidad y pertenencia, contribuyendo por defecto a la cohesión social. La integración social es especialmente importante en países como Chile, que han vivenciado experiencias históricas traumáticas, caracterizadas por largas dictaduras, la sistemática vulneración de derechos humanos y la violencia política, lo que ha agudizado la polarización y el enfrentamiento de grupos sociales que se perciben como adversos.

Atendiendo a esta problemática, si bien en Chile se han articulado esfuerzos importantes en materia educativa que revalorizaron la participación y la formación ciudadana como contenido transversal del currículum escolar, persisten deficiencias vinculadas a la instalación de competencias cívicas e interiorización de principios democráticos en el alumnado, así como también una fuerte estratificación por capas sociales de los resultados escolares en lo que a estas materias respecta. El Estudio Internacional sobre Educación Cívica y Ciudadanía (Schulz et al., 2009) puso en evidencia los déficits en conocimiento cívico y las grandes diferencias que se erigían con base al nivel socioeconómico. Otras investigaciones han relevado la importancia de avanzar en una democratización real de la cultura escolar (Muñoz, 2011b), reducir el distanciamiento entre los jóvenes y la política (Cox, Jaramillo y Reimers, 2005) y promover hábitos de participación electoral y de prácticas deliberativas (Díaz, 2014).

Naturalmente, el instrumento por el que la sociedad delega a la institucionalidad educativa el perfil de ciudadano que pretende formar lo constituye el currículum escolar. En él se imprime la visión del ciudadano que imagina, sus requerimientos cognitivos y morales, así como la memoria histórica que pretende legar (Bascopé, Cox y Lira, 2015). 
La experiencia chilena ha dado cuenta de diferentes modalidades de presentación de la educación cívica-ciudadana en el currículum escolar con el fin de profundizar sus alcances y significaciones en el estudiantado. En cuanto a contenido se ha visibilizado como una asignatura independiente, como porción de la Historia y las Ciencias Sociales y como objetivo transversal del currículum general. También se ha evidenciado un tránsito en la revalorización de los contenidos desde la Educación Cívica a la Educación Ciudadana, paradigma este último que supera la restricción de abordar la mera institucionalidad del Estado y que aporta una visión más integral de la ciudadanía al incorporar la importancia del contexto y la formación civil, ello sin desvincularse de la transmisión del conocimiento cívico.

En su análisis histórico, tanto la modalidad como los contenidos de ciudadanía operacionalizados en los curriculum escolares no han sido invariables en su cronología, ni asépticos respecto de los vertiginosos cambios políticos que han hecho resonancia en la sociedad. A juicio de Bascopé et al. (2015), en el contexto de la dictadura, específicamente a partir de la reformulación de los planes y programas de estudio en 1980, se constata una ideologización nacionalista del currículum cívico-ciudadano con fuerte énfasis en el conocimiento de las instituciones y en el fortalecimiento de la identidad patriótica. Por otra parte, el currículum del periodo de transición a la democracia se centraría, fundamentalmente y no obstante sus debilidades, en las competencias para la participación y en los valores cívico-democráticos tales como la cohesión, la equidad y la justicia social.

La participación estudiantil como expresión de los valores cívicos y democráticos que la formación ciudadana en esta nueva mirada curricular pretende instalar no está exenta de obstáculos. A este respecto, la dificultad para la constitución de orgánicas estudiantiles y los reducidos niveles de participación e injerencia en la toma de decisiones están dentro de las principales debilidades del currículum en materia de formación ciudadana y de organización estudiantil. Ello pese al esfuerzo desplegado por el movimiento estudiantil en el último tiempo para constituirse en un actor de relevancia en la contingencia educativa nacional, evidenciada en el rol protagónico que le correspondió cumplir en los procesos prerreforma educativa para instalar en la agenda las problemáticas de calidad, gratuidad y fin al lucro, elementos que constituyen las bases fundantes de la nueva política educativa abordada por el Estado de Chile a partir de 2012. Chile tiene formalmente constituido un sistema de medición de la calidad educativa (SIMCE), que traduce su accionar principalmente en la aplicación anual de pruebas estandarizadas que buscan determinar el nivel de aprovechamiento que los estudiantes chilenos hacen de los contenidos del currículum, limitación que la nueva reforma educativa busca superar, construyendo una institucionalidad que aborde la integralidad del hecho educativo, ampliando su ámbito de injerencia y medición hacia otros componentes otrora ignorados, tales como la convivencia escolar, los hábitos de vida y la participación ciudadana, entre otros. 
Pese a que la educación cívica-ciudadana ha evidenciado cambios en su estructura y contenido, no logra compensar por completo los déficits que, a más de veinticinco años del término de la dictadura cívico-militar, aun socaban la consolidación de la institucionalidad democrática chilena, requiriendo para estos efectos promover desde la praxis la participación en todas sus expresiones, la cohesión y el sentido de pertenencia, así como reconocer y dar voz a las orgánicas estudiantiles al amparo de una real experiencia de democracia.

La educación para la ciudadanía encierra un enorme potencial para mejorar la calidad de la democracia. El conocimiento cívico, la socialización política, la participación y la asimilación de actitudes prodemocráticas constituyen valiosos recursos para ejercer la ciudadanía, que posibilitarían corregir la magra calidad de la democracia y sus principales síntomas: la pérdida de confianza en las instituciones y la fuerte tendencia a la desafección política, sobre todo en las cohortes más jóvenes. De igual modo, la formación ciudadana incorpora competencias críticas que permiten mejorar cualitativamente las decisiones públicas y contribuir a generar mayores exigencias de la rendición de cuentas (accountability) social. Estos elementos adquieren suma importancia al considerar las problemáticas cada vez más evidentes de la coyuntura democrática chilena. Según datos de la Corporación Latinobarómetro, ${ }^{4}$ en Chile al año 2013 solo un 3,3\% de la población reportó estar muy satisfecho con el funcionamiento democrático del país, cifra que se aleja fuertemente del promedio latinoamericano (12,8\%). De igual forma, como fenómeno sociopolítico, llama la atención la marcada tendencia a la baja en el involucramiento en mecanismos formales de participación, tales como el sufragio, la militancia política y la filiación sindical. Eventos de desafección ciudadana que tienden a ser más notorios en la población joven y en grupos socioeconómicos bajos, situación que podría constituir una prolongación de las experiencias educativas con las que han sido formados.

En síntesis, la participación y la formación ciudadana al interior de la escuela emergen como una posible solución frente a los déficits de la calidad democrática del país, aunque no exenta de la dinámica reproductivista del sistema educacional chileno. Es por esto que el presente artículo busca caracterizar las acciones que desde la escuela se realizan en pos de potenciar la organización y participación estudiantil, el nivel de adhesión que estas registran en el estudiantado y el sentimiento de apego y pertenencia, así como las estrategias pedagógicas para desarrollar actitudes prodemocráticas en la escuela. Estos aspectos son abordados desde la segmentación socioeducativa que la institucionalidad escolar da lugar, ello a partir de la comparación de un conjunto de índices estandarizados y estadísticos porcentuales en una muestra censal de estudiantes de $8^{\circ}$ año básico.

\footnotetext{
${ }^{4}$ La Corporación Latinobarómetro lleva a cabo anualmente encuestas individuales de opinión pública en asuntos políticos, económicos y sociales de dieciocho países latinoamericanos. Bases de datos en http://www.latinobarometro.org/
} 


\section{METODOLOGÍA}

La Agencia de Calidad de la Educación (ACE), organismo dependiente del Ministerio de Educación de Chile, aplica anualmente cuestionarios estructurados de contexto que exploran diversas dimensiones del desarrollo sociopersonal del estudiantado. En su conjunto estas dimensiones han recibido el nombre de Otros Indicadores de Calidad Escolar (OIC), a los que recientemente se les ha asignado una ponderación dentro del proceso de clasificación de escuelas en categorías de desempeño (Alto, Medio, MedioBajo, Insuficiente). Entre estos nuevos indicadores se ha considerado la medición de la Participación y Formación Ciudadana, que evalúa en qué medida el establecimiento promueve el Sentido de Pertenencia, la Participación y el desarrollo de habilidades y actitudes para la Vida en Democracia. Este proceso de relevamiento de información tiene una cobertura censal a nivel de estudiantes, apoderados y profesores.

El presente estudio utiliza la base de datos correspondiente al cuestionario de contexto para estudiantes del año 2013, centrando su interés en la dimensión de Participación y Formación Ciudadana. Este cuestionario se aplica a nivel nacional a todos los estudiantes que están finalizando la enseñanza básica en algún establecimiento educativo reconocido por el Estado de Chile, el que según su dependencia administrativa puede ser de carácter público (municipal), privado con financiamiento estatal (particular subvencionado) o particular pagado.

La muestra agrupa a un conjunto de 216.158 estudiantes de $8^{\circ}$ básico, de los que un $50,4 \%$ son hombres. La media y mediana de edad fueron 13,72 y 14 respectivamente. La distribución por dependencia educativa indica que un $42,4 \%$ de los estudiantes proviene del sistema de enseñanza municipalizado, un $49,8 \%$ del particular subvencionado y un $7,8 \%$ del particular pagado.

La investigación es de tipo no experimental, de corte transversal y de carácter cuantitativa. El análisis de los datos opera a nivel descriptivo mediante la comparación de índices ad hoc y estadísticas descriptivas con base en la dependencia educativa de los escolares.

El procesamiento de los datos mediante el uso del software estadístico SPSS versión 21 contempló la construcción de los siguientes índices estandarizados en un rango 0-1:

- Vida Democrática: dimensión subdividida en tres componentes a razón de cuatro ítems cada uno. Los dos primeros exploran la existencia, modalidad de organización y participación en la orgánica estudiantil a nivel de curso (Actividad Democrática en Consejo de Curso) y colegio (Actividad Democrática en Centros de Alumnos). Por otra parte, el componente Promoción Docente a la Actividad Democrática aborda un conjunto de tareas y estrategias pedagógicas implementadas por el docente para desarrollar habilidades y actitudes prodemocráticas. 
- Sentido de Pertenencia: índice constituido por cinco ítems con el objetivo de dimensionar el nivel de apego que vivencia el estudiante respecto de la institución escolar a la que está formalmente adscrito.

- Participación: con base en la existencia de actividades de distinta naturaleza efectuadas en y por el establecimiento, este índice busca cuantificar el nivel de adhesión del estudiantado en cuanto a participación y colaboración en la realización de estas actividades.

\section{ANÁLISIS}

\section{Dimensión de Vida Democrática}

En sintonía a lo que plantean Cox y Castillo (2015), no nacemos demócratas, sino que aprendemos a serlo. En este sentido, la escuela se constituye en un espacio privilegiado para intencionar las competencias participativas, deliberativas, críticas y de escucha activa que componen la piedra basal de los sistemas democráticos exitosos, validando a la escuela como un escenario idóneo para el desarrollo de una "comúnunidad" de construcción de significados éticos (Redon, 2010).

En atención a ello se visualiza que en términos formales se ha instalado un sistema de participación y promoción para la vida democrática en la escuela. Los alumnos informan el desarrollo de una Actividad Democrática en los Consejos de Curso (0.78) y una Actividad Democrática en los Centros de Alumnos (0.72). Igualmente relevante, pero en una proporción menor, se encontraría el conjunto de acciones docentes de Promoción a la Actividad Democrática en los estudiantes. En términos globales, este componente promedia en 0,71 puntos y se ubica como el de menor puntuación de la dimensión analizada, alcanzando diferencias de hasta un 10\% inferior a la obtenida por los otros componentes del índice, no obstante esta diferencia debe ser considerada con cautela toda vez que su puntuación es moderada a la baja por el accionar especifico de uno de sus ítems (Organizan Debates), que registra una presencia comparativamente más marginal.

En términos formales se constata la presencia de Centro de Alumnos (84.2\%) y de Consejo de Curso (71.7\%), espacios donde los alumnos tendrían la posibilidad de elegir y ser elegido, así como de organizarse para enfrentar problemáticas que les son afines. En sintonía a lo planteado por Marshall y Bottomore (1998) éstos tributarían a la esfera política de los derechos ciudadanos. 
Tabla 1: Índices de Vida Democrática por tipo de establecimiento

\begin{tabular}{|c|c|c|c|c|}
\hline & Municipal & Part. Subv. & Part. Pagado & Total \\
\hline Actividad democrática en Centro de Alumnos & $x=0.71$ & $x=0.71$ & $x=0.84$ & $x=0.72$ \\
\hline Existencia de Centro de Alumnos & 83.5 & 83.2 & 93.2 & 84.2 \\
\hline Elegido por votación de los* estudiantes & 84.9 & 86.9 & 94.3 & 86.8 \\
\hline Información candidatos y programas* & 71.9 & 77.3 & 88.5 & 76.2 \\
\hline Participación electoral ${ }^{\star}$ & 63.8 & 62.2 & 74.1 & 63.9 \\
\hline Actividad democrática en Consejo de Curso & $x=0.76$ & $x=0.79$ & $x=0.79$ & 0.78 \\
\hline Consejo de Curso semanalmente & 68.5 & 73.7 & 76.4 & 71.7 \\
\hline Es dirigido por la directiva & 73.9 & 77.3 & 75.6 & 75.7 \\
\hline Se tratan temas de interés & 75.7 & 76.6 & 75.1 & 76.1 \\
\hline Elegido por votación popular & 87.6 & 89.4 & 90.6 & 88.7 \\
\hline Promoción Docente a la actividad democrática & $x=0.71$ & $x=0.71$ & $x=0.68$ & 0.71 \\
\hline Promueven la participación & 82.3 & 84.8 & 86.4 & 83.8 \\
\hline Estimulan a la opinión & 83.6 & 84.3 & 79.6 & 83.6 \\
\hline Promueven la escucha y el respeto a las otras opiniones & 88.3 & 90.5 & 91.1 & 89.6 \\
\hline Organizan debates & 55.0 & 49.3 & 39.4 & 51.0 \\
\hline
\end{tabular}

Fuente: Elaboración propia con base en cuestionarios de contexto SIMCE 2013

Se constata en el componente Actividad Democrática en Centros de Alumnos una diferencia significativa entre la información que reportan los estudiantes de colegios Particulares pagados (0.84) respecto de sus pares de colegios Municipales (0.71) y Subvencionados (0.71). Diferencia que se observa sistemáticamente en cada indicador evaluado, siendo más aguda y preocupante en los ítems de Información de Candidatos y Programas y Participación Electoral.

En la Tabla 1 se observa que el $84.2 \%$ de los estudiantes reportan la Existencia de un Centro de Alumnos (CCAA) en su unidad educativa, no obstante evidenciar ciertas diferencias al desagregar este porcentaje por tipo de establecimiento. Esta información es consistente con el estudio de Unicef (Lafferte y Silva Gallinato, 2014), donde se advierte que el problema de la participación estudiantil en el contexto escolar no es la ausencia de CCAA, sino el déficit de representatividad e involucramiento en ellos. En efecto, si bien la existencia de CCAA parece estar instalada transversalmente, el indicador no da cuenta de las deficiencias de carácter estructural vinculadas a las orgánicas estudiantiles, en particular el escaso peso resolutivo dentro de los Concejos Escolares u otras instancias de toma de decisiones (Sarramona y Rodríguez, 2010), así como la baja capacidad para alcanzar objetivos que le son atingentes. 
Si bien tan solo el $15.8 \%$ de los estudiantes reportan no tener una orgánica propia, este porcentaje se acrecienta significativamente al considerar su representatividad formal, esto es, que sean organizaciones elegidas por escrutinio popular. El indicador Elegido por votación de los estudiantes evidencia porcentajes relativamente altos (86.8\%), aunque conservando una lógica de segmentación que favorece a los colegios particulares pagados por sobre las otras dependencias educativas. Sin embargo, pese a constituir un indicador de positivas cifras, no se puede pasar por alto que el $15.1 \%$ y el $13.1 \%$ de los estudiantes de establecimientos Municipales y Particulares Subvencionados, respectivamente, reportan que los CCAA fueron elegidos por modalidades diferentes a la votación popular. Situación que, entre líneas, podría interpretarse como una imposición de los representantes escolares por parte de directivos, profesores o autoridades en general, infringiendo principios democráticos básicos, aun cuando los reglamentos ministeriales fijan parámetros que hacen de la votación de CCAA una actividad universal, unipersonal, secreta e informada.

Respecto del indicador de Información de Candidatos y Programas, un 68\% de los estudiantes reportaron haber accedido a este tipo de información antes de la elección del CCAA, aunque al desagregar el análisis por tipo de establecimiento se registra un cambio porcentual del $23 \%$ a favor de los colegios particulares pagados respecto de los municipales. Sesgo que instala una problemática en torno al voto informado, toda vez que, para tomar decisiones de esta naturaleza, los estudiantes deben tener acceso al total de la información disponible por los canales institucionales correspondientes, asegurando así que sus intereses se vean representados. Situación de alta importancia en atención al contexto de apatía y desafección político-electoral que se ha ido instalando progresivamente durante las últimas décadas en la sociedad chilena y que podría ser revertido a partir de la promoción de experiencias democráticas al interior de las escuelas, especialmente si van acompañadas de un énfasis en el rol que juega la información en las responsabilidades de monitoreo y participación ciudadana.

De acuerdo con el indicador de Participación Electoral se observa que un $42 \%$ de los estudiantes no votó en las elecciones del Centro de Alumnos en su establecimiento, ya sea porque no se registra la presencia de CCAA o bien existiendo se marginan de participar en esta decisión (36,1\%). Preocupante cifra que se condice con las bajas tasas de sufragio juvenil, agudizada por la instauración de la voluntariedad del voto en elecciones de representantes políticos, sean del presidente, congresistas o alcaldía (Contreras y Morales, 2014). Igual de preocupante resulta la segmentación de la participación electoral según el tipo de establecimiento, donde se constata una diferencia relativa que posiciona a los establecimientos Particulares Pagados en un $16 \%$ por sobre los Municipales. Naturalmente, si la desafección electoral se evidencia durante el periodo escolar es muy probable que se replique una vez que egresen del colegio, deteriorando la calidad de la democracia. Tal como señala 
Corvalan y Cox (2015), cuando la propensión a votar correlaciona con características no aleatorias de la población se induce directamente un problema de representatividad, o sesgo de clase, lesionando el principio de igualdad democrática. Se vulnera en la práctica el precepto sobre el que se fundan y consolidan las democracias modernas, en tanto que todos los ciudadanos gozan de mismos derechos y obligaciones a pesar de la desigualdad de las circunstancias y condiciones que les toca vivir. Por esta razón, como consecuencia de la marginación en la intención de voto de un determinado conglomerado, se subrepresentan sus demandas y necesidades en el marco institucional de la vida democrática

Si bien, puede establecerse a nivel predictivo cierta sintonía entre la apatía juvenil y la desafección futura que se tendría respecto los procesos político-sociales, se reconoce que este desinterés se posiciona con más notoriedad en la esfera institucional o de la participación formal (Vargas, Oyanedel y Torres, 2015). En consecuencia, existiría un conjunto de nuevas manifestaciones de comportamiento y compromiso ciudadanos de los jóvenes invisibilizado, que sería expresión de otras prácticas participativas y de construir en común-unidad lo público, constituyéndolos como sujetos políticos que ejercen ciudadanía en sus diversos ámbitos de interacción (Agudelo, Murillo, Echeverry y Patiño, 2013). Espacio de cotidianidad escindido de la institucionalidad formal que, al ser compartido con otros, implica un necesario compromiso ético.

No obstante lo anterior, dentro de la institucionalidad formal la organización estudiantil por antonomasia en la escuela es el Consejo de Curso. Aunque los CCAA presentan una visibilidad pública mayor en razón a su condición de órgano estudiantil de nivel superior e inclusión en el Consejo Escolar, ${ }^{5}$ es en los Consejos de Curso donde se constata una mayor tradición de participación. A nivel estudiantil son estas organizaciones de base donde los alumnos adquieren, no obstante sus bemoles, las primeras experiencias democráticas, donde se manifiestan los incipientes roles dirigenciales y emergen las características de liderazgo, además de ser la primera instancia donde se ponen en común los temas que les son atingentes. Atributos destacables de los Consejos de Curso, a pesar de no registrar la misma ceremonia y protocolo característico de la elección, constitución y sesionar de los CCAA. En efecto, el acto eleccionario es más informal, se realiza a mano alzada, rara vez se cuenta con espacio donde los eventuales candidatos den a conocer sus propuestas, e incluso las sesiones se realizan con presencia del profesor, a quien le asiste el derecho a intervenir de considerarlo necesario, lo que puede restar autonomía a la organización misma. Bajo estas condiciones es natural que la participación electoral sea alta, pero no por ello exenta de problemas de legitimidad y efectividad. En efecto, se constituyen

${ }^{5}$ El Consejo Escolar es un órgano institucional de constitución obligatoria en escuelas municipales y subvencionadas que está integrado por representantes de los directivos del establecimiento, sostenedores, profesores, asistentes de la educación, padres o apoderados y estudiantes. 
mayoritariamente mediante elección popular $(88.7 \%)$ y en cifras muy similares a las encontradas en la conformación de los CCAA (86.8\%). Modalidades distintas y reñidas con la representatividad democrática, tal como lo sería la designación o intervención de autoridades y docentes, tienen una presencia atenuada que bordea el $11 \%$.

Los problemas vinculados a los Consejos de Curso no estarían determinados por su modalidad de constitución sino básicamente por su quehacer como orgánica estudiantil. Tan solo un $76,1 \%$ de los estudiantes reconoce que se tratan en ellos temas de interés, un $75,7 \%$ que son dirigidos por la directiva y un $71,7 \%$ que opera semanalmente. Ítem este último que, a diferencia de los anteriores, muestra contrastes significativos según la dependencia del establecimiento, que puede alcanzar una variación porcentual del orden del $11 \%$ en detrimento de los colegios Municipales.

Se informa una falta de periodicidad en el sesionar de los Consejos de Curso, en especial en los colegios Municipales, donde tan solo un $68,5 \%$ de los estudiantes reconoce un funcionamiento semanal. Los Consejos de Curso a nivel de enseñanza básica, contrario a lo que acontece en la enseñanza media, no tienen un espacio único y con carga horaria asignada en los currículums que conforman los planes y programas de cada nivel. Esta actividad es compartida con el sector de Orientación, donde cada unidad educativa define las estrategias que empleará a fin de desarrollar los objetivos que de ella se demandan acorde a los propósitos de los respectivos proyectos educativos institucionales y la organización interna de los establecimientos.

De esta manera, algunas unidades educativas optan por programar las sesiones del Consejo de Curso dentro de las horas de libre disposición o bien hacer uso de un tiempo compartido con las horas de Orientación, asignándole una porción de su tiempo semanalmente, mientras que otros establecimientos optarían por la modalidad de rotación quincenal. Esta estructuración curricular resta autonomía relativa a la orgánica estudiantil de los Consejos de Curso, situación que se devela en que uno de cada cuatro estudiantes informa que éstos no son dirigidos por la directiva $(24,3 \%)$, sin diferencias importantes por tipo de establecimiento. Al no disponer de un tiempo de administración propio se corre el riesgo de otorgar al profesor un excesivo protagonismo, interviniendo en problemáticas y decisiones que pueden no estar en sintonía con las prioridades y demandas que los propios estudiantes manifiestan y de las que, como miembros del grupo del curso, deben hacerse responsables.

Si bien el acompañamiento del docente en temas relativos al Consejo de Curso pierde progresivamente importancia conforme se transita a niveles más altos de enseñanza, que por lo demás se acompañan de una mayor autonomía estudiantil, su rol como formador y promotor de la participación y la democracia se mantiene vigente y transversal a las prácticas pedagógicas con que actúa, sobre todo si en sus metodologías de aula se interiorizan valores intrínsecamente democráticos, tales como la escucha activa, la valoración de la opinión, la negociación y el diálogo.

En consecuencia, en la Tabla 1 el componente de Promoción Docente captura una tenue diferencia de medias según la dependencia del establecimiento que sitúa a 
las escuelas Municipales y Particulares Subvencionadas 0.03 puntos en la escala estandarizada de 0 a 1 , por sobre los establecimientos Particulares pagados $[F(2$, $212.658=134,158 ; p<0,001)$ ]. Desagregada esta dimensión en los ítems que la componen, se constata que las actividades docentes que promueven la participación realizada con mayor frecuencia (Siempre o Casi siempre) está determinada por incentivar la escucha activa y respetuosa de las opiniones de sus pares (89.6\%) y promover la participación estudiantil dentro de la sala de clases (83.8\%), estimulando la expresión de ideas, juicios y opiniones (83.6\%). Ítems que por lo demás no registran grandes diferencias con base en el tipo de establecimiento.

La acción de promoción a la vida democrática más infrecuente es la organización de debates escolares, donde tan solo el $51 \%$ de los estudiantes reporta su realización Siempre o Casi siempre. Ítem donde se registran las mayores diferencias entre unidades educativas, siendo especialmente significativa su ausencia como estrategia pedagógica en los colegios Particulares pagados. A diferencia de las otras acciones de promoción para la vida democrática contemplada en este índice, la organización de debates implica, por parte del docente, una planeación más detallada y consciente en cuanto a técnica didáctico- pedagógica, escapando de las estrategias tradicionales de enseñanza utilizadas en aula por el profesor. Práctica innovadora que precisa de profesores y de alumnos mayores exigencias de organización y tiempo, requerimientos que no siempre se condicen con las otras demandas pedagógicas.

La ausencia de orgánica estudiantil, su falta de representatividad en atención a su constitución y accionar, los deficientes procesos de elección popular conocida e informada, así como otras debilidades vinculadas a la promoción para la vida democrática en la escuela serían expresión de un proceso gradual y generalizado de desafección política y ciudadana, al menos en lo que a participación formal refiere, cuyas manifestaciones se vivencian también en la institución educativa. Existirían restricciones institucionales implícitas o explícitas que se instalan por parte del sostenedor o cuerpo directivo, quienes no brindarían los espacios para el debate y la exposición de ideas entre los estudiantes.

La acción y discusión democrática en la escuela es visualizada por la autoridad como una eventual transgresión a los tiempos lectivos que vulnera a las ya rigurosas planificaciones curriculares. A ello se suma un dejo de desconfianza en otorgar espacios de autonomía a la organización estudiantil, pues siempre está presente el riesgo de que, en un proceso de empoderamiento natural, se escale en las demandas y reivindicaciones que pudiesen ser antagónicas a la gestión administrativa y curricular vigente.

Complementario a lo anterior, se encontraría la presión de las familias por restringir la participación política en los establecimientos, considerándola una temática sensible o no pertinente con los sellos ideológicos familiares, etiqueta que también reciben otros tópicos como la sexualidad (Mardones, 2015). No son pocas las familias que consideran la participación política estudiantil e incluso el abordaje de 
determinados temas de contingencia política en la escuela como algo preocupante y perjudicial para la formación de sus hijos.

En consecuencia, tanto la participación política de sus hijos como la promoción de actividades de formación para la vida democrática son concebidas como acciones escindidas de lo estrictamente pedagógico y ajenas a la responsabilidad que le compete a la escuela, por cuanto persistiría la creencia que los niños y niñas al estar en proceso de formación son vulnerables a la inculcación maliciosa que puedan hacer determinados docentes, proporcionando interpretaciones no solo sesgadas de lo político sino además en conflicto directo con los marcos conceptuales e ideológicos en que se manejan los padres.

\section{Dimensión Sentido de Pertenencia}

Con la instalación del modelo neoliberal en Chile y la competencia como su estandarte se ha observado una atomización de las estructuras colectivas relegadas al resultado aditivo, más no sinérgico, de los individuos que la componen. Esta es una tendencia de fragmentación social que, entre otras consecuencias, implicaría una disolución de los lazos e identidades colectivas, una pérdida de confianza en lo público y un debilitamiento del sentido de pertenencia (Cox et al., 2005).

Estos aspectos son también constatados en la escuela y pueden socavar aún más la cohesión social. La segmentación socioeducativa profundiza la estratificación territorial, inhibiendo la capacidad de la escuela de constituirse en un espacio de convivencia en el que convergen grupos de capital social, cultural y económico diferenciado, perdiendo así el atributo de ser máquina antiliberal especialista en reciprocidad (Antelo, 2007).

De acuerdo con Redon (2010) el sentido de pertenencia implica por parte del alumno compartir los valores, preferencias, ideologías y logros que el establecimiento educativo persigue, así como sentir que el lugar donde estudia le es propio. Este sentido de pertenencia con el entorno es vital para el desarrollo de la identidad y autoestima personal, a la vez que constituye una condición necesaria para el ejercicio efectivo de la participación. Además, Mieles y García (2010) postulan que el respeto y la valoración de la pluralidad derivan del hecho de pertenecer a un grupo y de hacer efectiva la aceptación mutua con otros grupos de características distintas. 
Tabla 2: Índice de Sentido de Pertenencia según tipo de establecimiento

\begin{tabular}{|l|c|c|c|c|}
\hline & Municipal & Part. Subv. & Part. Pagado & Total \\
\hline Sentido de Pertenencia & $\mathbf{0 . 6 9}$ & $\mathbf{0 . 6 7}$ & $\mathbf{0 . 7 1}$ & $\mathbf{0 . 6 8}$ \\
\hline Ir contento al establecimiento & 47.2 & 46.6 & 59.5 & $\mathbf{4 7 . 8}$ \\
\hline Orgulloso por el Establecimiento & 85.4 & 82.4 & 85.0 & 83.9 \\
\hline Hablo bien del establecimiento a otras personas & 83.4 & 80.2 & 83.0 & 81.8 \\
\hline Recomendaría el establecimiento & 69.3 & 63.4 & 68.0 & 66.2 \\
\hline Defendería el establecimiento ante los demás & 74.7 & 74.5 & 76.5 & 74.8 \\
\hline
\end{tabular}

Fuente: Elaboración propia con base a cuestionarios de contexto SIMCE 2013

Con base en la Tabla 2 se observa en el índice estandarizado un debilitamiento general del sentido de pertenencia (0.68) que, inclusive en términos comparativos, alcanza una cifra inferior al resto de las escalas analizadas. Índice que es moderado a la baja principalmente por efecto del primer ítem, donde se reporta que más del $50 \%$ de los alumnos no asisten contentos a sus establecimientos. Desmotivación y desinterés que tienden a ser menores en el segmento de estudiantes de colegios particulares, donde la procedencia socioeconómica familiar hace que la predisposición al aprendizaje por parte del alumno y las condiciones del ambiente educativo sean eventualmente mejores. En efecto, al comparar por tipo de establecimiento, en el primer ítem se constatan cambios porcentuales de $21 \%$ y $22 \%$ entre los estudiantes de colegios particulares pagados y los estudiantes de colegios municipales y subvencionados respectivamente.

Más allá de la diferencia expresada anteriormente, el índice estandarizado, así como el desglose del resto de sus ítems, no logra capturar diferencias sustantivas por tipo de establecimiento, comportamiento que llama la atención. Pese a que los estudiantes mayoritariamente declaran no ir contentos a sus establecimientos, sienten orgullo de pertenecer y le atribuyen características favorables al hablar de su colegio ante los demás.

El acto educativo expresado en el hecho de tener que ir al colegio tiende a ser asumido, en especial a esta edad, como una obligación instigada a nivel familiar e institucional. Obligatoriedad que tiende a despertar el malestar de asistir al colegio frente al tedio de la conducta rutinizada, a la que se le suma la existencia de un espacio institucional que le es hostil y ajeno. Las escuelas con bajo nivel de logro o de convivencia deteriorada tienden a manifestar mayores dificultades al momento de acoger a sus alumnos. Situaciones de violencia escolar y de bullying en particular hacen que el alumno se sienta inseguro y asista con miedo al colegio. Frente a esta situación no son pocas las instituciones que optan por aumentar sus medidas de seguridad, así como padres que lo demandan. 
Si bien la identidad común que provee la vida compartida es indispensable para tejer lealtades entre la comunidad y adquirir compromisos cívicos básicos para el funcionamiento de la democracia (Peña, 2007), el sentido de pertenencia puede no visibilizar los fenómenos de polarización social que han sido característicos de las sociedades latinoamericanas. Es decir, los escolares, en cuanto a grupo social, podrían sentirse muy identificados con miembros de su propio subconjunto pero distantes respecto de otros (Gasparini y Molina, 2006), de manera que el sentido de pertenencia con la institución educativa no es necesariamente una garantía de la cohesión social. Expresión de lo anterior es el hecho que con independencia del tipo de establecimiento los alumnos reportan altos niveles de adhesión institucional, sintiendo orgullo de formar parte de la unidad educativa (83.9\%), refiriéndose positivamente sobre ella (81.8\%) y defendiéndola ante los demás (74.8\%), todo ello aun cuando la calidad y pertenencia social entre los establecimientos estén profundamente segmentadas. En efecto, no es posible esperar un genuino sentido de pertenencia escolar si la diversidad cultural y la mixtura social no forman parte de la ecuación en la institucionalidad educativa. Los estudios muestran que en el acto de segregación no solo se restringen las oportunidades educativas de los grupos más desfavorecidos (Ortiz, 2015) sino que emerge el prejuicio, el rechazo y el miedo a la diferencia (Bellei, Canales, y Orellana, 2015). Situación que pone en entredicho el rol del espacio escolar, que es instalar también las habilidades necesarias e imprescindibles para vivir en comunidad. La escuela ha sido una institución particularmente exitosa en su capacidad para regular las proximidades, dosificar los desprecios y enseñar el arte de tolerar a los otros (Antelo, 2007), función que en el segmentado contexto educativo nacional se encuentra profundamente limitada.

\section{Dimensión de Participación}

La participación ciudadana juega un rol importante en el proceso de modernización del Estado. Desde un punto de vista instrumental la participación pasa a ser un fuerte mecanismo de control político y rendición de cuentas (accountability) que reconoce el potencial de la ciudadanía organizada para mejorar las gestiones y decisiones públicas. Esta influencia o capacidad para redefinir las prioridades de la agenda pública constituye a su vez un fin en sí mismo que es expresión del más genuino espíritu de la democracia. De acuerdo con Rivera y Santos (2015), sin participación el sistema democrático pierde legitimidad. 
Tabla 3: Índice de Participación según tipo de establecimiento

\begin{tabular}{|c|c|c|c|c|c|c|c|c|}
\hline \multirow{3}{*}{$\begin{array}{l}\text { Participación en actividades } \\
\text { en el establecimiento }\end{array}$} & \multicolumn{2}{|c|}{ Municipal } & \multicolumn{2}{|c|}{ Part.Subv. } & \multicolumn{2}{|c|}{ Part. Pagado } & \multicolumn{2}{|c|}{ Total } \\
\hline & \multicolumn{2}{|r|}{0.55} & \multicolumn{2}{|r|}{0.54} & \multicolumn{2}{|r|}{0.58} & \multicolumn{2}{|r|}{0.55} \\
\hline & S/MV & NO & S/MV & NO & S/MV & NO & S/MV & NO \\
\hline Recreativas & 52.7 & 6.3 & 53.3 & 6.2 & 63.8 & 3.1 & 53.9 & 6.0 \\
\hline Deportivas & 52.7 & 3.8 & 46.1 & 5.1 & 56.3 & 2.3 & 49.7 & 4.3 \\
\hline Académicas y culturales & 37.0 & 9.2 & 37.2 & 8.7 & 40.0 & 4.6 & 37.4 & 8.6 \\
\hline Campañas solidarias & 48.5 & 7.6 & 47.8 & 8.2 & 51.3 & 5.0 & 48.4 & 7.7 \\
\hline Inicio y cierre de períodos académicos & 68.1 & 2.7 & 70.7 & 2.1 & 69.6 & 2.0 & 69.5 & 2.3 \\
\hline Conmemorativas & 73.4 & 3.1 & 74.8 & 2.4 & 77.8 & 1.6 & 74.4 & 2.6 \\
\hline \multirow{2}{*}{$\begin{array}{l}\text { Colaboración en la realización } \\
\text { de las actividades del establecimiento }\end{array}$} & \multicolumn{2}{|r|}{0.48} & \multicolumn{2}{|r|}{0.44} & \multicolumn{2}{|r|}{0.43} & \multicolumn{2}{|r|}{0.45} \\
\hline & S/MV & NO & S/MV & NO & S/MV & NO & S/MV & NO \\
\hline Recreativas & 56.8 & 2.7 & 51.0 & 3.3 & 47.9 & 2.7 & 53.2 & 3.0 \\
\hline Deportivas & 55.4 & 2.1 & 47.2 & 3.1 & 48.5 & 1.7 & 50.8 & 2.5 \\
\hline Académicas y culturales & 36.9 & 6.7 & 33.3 & 7.1 & 32.9 & 3.6 & 34.8 & 6.7 \\
\hline Campañas solidarias & 41.6 & 8.0 & 38.3 & 8.3 & 40.8 & 4.1 & 39.9 & 7.9 \\
\hline Conmemorativas & 44.5 & 5.7 & 41.0 & 5.7 & 37.5 & 4.9 & 42.2 & 5.6 \\
\hline
\end{tabular}

Nota: $\mathrm{S} / \mathrm{MV}=$ Siempre /la mayoría de las veces. No = No se organiza

En su origen etimológico participar significa tomar parte de algo, habitualmente una cuota de poder en el proceso decisorio (Cohen y Franco, 1992). Esta definición aplicada al espacio escolar se traduciría en que las decisiones en asuntos que afectan directamente a los estudiantes son resultado del diálogo, la negociación y la valoración de las opiniones, situación que posibilitaría adquirir competencias básicas de compromiso cívico, actitudes que se prolongarán en su vida adulta (Sarramona y Rodríguez, 2010).

La valoración de la participación en la retórica escolar contrasta con la realidad educativa nacional. Diversos investigadores, tales como Muñoz (2011a) y Redon (2010), identifican un debilitamiento de prácticas deliberativas y participativas expresadas en el ejercicio espurio de la toma de decisiones, las que son relegadas a una participación de carácter informativa o a la manipulación de variables en asuntos minúsculos del ámbito escolar. Misma situación es identificada en otras latitudes latinoamericanas, donde se constata que, si bien existen marcos reglamentarios que protegen y crean órganos de participación, la incidencia en las estructuras de poder y toma de decisiones ha sido más bien escasa, restringiendo la participación estudiantil a 
instancias lúdicas, culturales y deportivas (Vergara, Montaño, Becerra, León-Enríquez y Arboleda, 2011).

Dado los ítems que incluye la ACE en la medición de la Participación es difícil sostener que capture como rasgo el nivel de codecisión de los estudiantes en los temas que les son de interés. Más bien, la medición de la Participación, con base en los instrumentos de contexto, queda relegada al acceso de los estudiantes a diferentes espacios de encuentro de la comunidad escolar, habitualmente programados por la escuela, ya sea en forma de actividades curriculares de libre elección o como actividades esporádicas.

Atendida esta limitación, la dimensión de Participación está conformada por dos índices estandarizados que buscan determinar la frecuencia con que los alumnos participan en las actividades del establecimiento y colaboran en la organización de las mismas. Así medida, esta dimensión carece de sensibilidad para determinar el nivel de codecisión de los estudiantes en sus asuntos de interés, toda vez que no pesquisa su real involucramiento y capacidad de injerencia en la toma de decisiones.

A nivel general, las actividades donde los alumnos participan con mayor frecuencia tienen relación con las de carácter conmemorativo (fiestas patrias, ceremonias religiosas) y las de inicio y cierre de periodos académicos. En este tipo de actividades siete de cada diez estudiantes informan participar siempre o la mayoría de las veces, sin constatarse grandes diferencias por tipo de colegio. La alta adhesión y escasa diferenciación por dependencia administrativa estarían determinadas por la naturaleza misma de la actividad, que al estar institucionalizada se constituye en un ritual de tránsito obligatorio, en el que la capacidad de decisión por parte del estudiante es reducida, cuando no inexistente.

Las otras actividades apelan en cambio a la voluntariedad, donde se develarían los intereses y habilidades individuales que conminan a formar parte de ella. Motivos que posibilitarían diferenciados niveles de participación con base en la naturaleza de la actividad consultada, siendo las Académicas-Culturales las que menos adhesión generan (37.4\%). A su vez, son estas mismas actividades no institucionalizadas las que registran mayores diferenciaciones por dependencia administrativa, siendo las Deportivas y Recreativas donde se constatan los mayores deltas, siempre a favor de los colegios particulares pagados. La variedad y diversificación de actividades extraprogramáticas tienden a ser un sello de distinción de la oferta particular pagada, con la que estos establecimientos pretenden diferenciarse en el mercado educativo de la competencia, evidenciando a su población objetivo una mayor preocupación por la formación integral, donde las actividades deportivas y recreativas le son consustanciales. Valor agregado tradicional que explicaría los mayores niveles de participación exhibidos en este tipo de colegios.

Por otra parte, respecto de la cooperación en actividades escolares se registra una mayor frecuencia de adhesión en las Deportivas y Recreativas. Las Actividades Académicas-Culturales no tan solo involucran una menor colaboración estudiantil, sino 
que además, junto con las Campañas solidarias, tienden a ser las que con menor frecuencia se realizan. Comparada por dependencia administrativa, es en los establecimientos municipales donde se contempla una mayor colaboración estudiantil, en especial en la realización de las actividades de corte recreativo o deportivo.

El bajo nivel de cooperación estudiantil en la organización de actividades observadas en los colegios particulares pagados estaría explicado porque en este tipo de establecimientos existe una mayor tendencia a gestionar institucionalmente tanto las actividades lectivas como no lectivas, disponiendo de los recursos humanos y organizacionales necesarios para tal efecto. Actividades planificadas y gestionadas desde el cuerpo directivo que, dejando menos espacio para la improvisación, contemplan niveles acotados de cooperación del estudiantado en su realización. Estas restricciones administrativas en establecimiento de naturaleza pública estarían menos presentes, por lo que la organización de actividades a ser ejecutadas solo se realiza de mediar la participación del estudiantado, dada la crónica estrechez de recursos humanos existentes.

\section{CONCLUSIONES}

La importancia de estudiar la segmentación de la Participación y la Formación Ciudadana consiste en ofrecer una proyección de las pautas de comportamiento civil y cívico de los estudiantes al momento de egresar de la unidad educativa. Acción pedagógica de relevancia que se instala en la escuela, con independencia relativa si su irrupción obedece a una consciencia de valor educativo intrínseco que la educación para la ciudadanía reporta, o bien se asume como una respuesta coyuntural a los nuevos tiempos, caracterizados por una profunda desafección que tendrían los jóvenes respecto de la organización social, el funcionamiento de la nación y la participación política. Sea como fuere, la escuela como institución educativa no puede soslayar su capacidad para ser agente que logra movilizar el sentido de justicia, tolerancia e igualdad, y demás virtudes ciudadanas que hacen posible vivir en una común-unidad.

Si bien se asume que el compromiso e interés en cuanto a sujetos no se agota ni limita exclusivamente a la esfera de la formalidad, existiendo un conjunto de comportamientos prosociales invisibilizados por la institucionalidad, en lo que a los datos respecta esta investigación muestra la existencia de diferencias en los índices de Participación y Formación Ciudadana con base en el tipo de dependencia educativa de los estudiantes. No obstante, las oscilaciones observadas son bastante menores a las que a priori podrían esperarse dada la segmentación estructural del sistema educativo y los antecedentes sociopolíticos de la población juvenil. Esta discrepancia, a juicio de los autores, deriva de la sensibilidad de los instrumentos de medida que emplea la ACE, los que podrían estar invisibilizando las expresiones más duras de la estratificación social en temas de participación y formación ciudadana. 
En cuanto a la dimensión de Vida Democrática se evidencia una segmentación en su componente de Actividad Democrática en los CCAA a favor de los estudiantes de colegios particulares pagados, aspecto que se hace aún más evidente en el ítem de participación electoral. No se constatan grandes diferencias en los componentes de Actividad Democrática en los Consejos de Curso y Promoción Docente de la Actividad Democrática, aunque en la desagregación por ítems de este último componente se observan estrategias de promoción diferenciales. Por ejemplo, la Organización de Debates, si bien es percibida transversalmente como una actividad con baja frecuencia, su representación en establecimientos municipales es significativamente más alta.

La dimensión de Sentido de Pertenencia expresa una puntuación diferencial en favor de los estudiantes de colegios particulares pagados que estaría explicada básicamente por la presencia del ítem Ir contento al establecimiento. Más allá de esta diferencia de motivación académica, en cualquier dependencia los estudiantes sienten orgullo de pertenecer a su colegio y le atribuyen características favorables al hablar de él ante los demás.

Respecto de la dimensión de Participación, los datos sugieren que el acceso a espacios de socialización de diversa naturaleza (recreativos, deportivos, culturales) se diferencia conforme a la demanda, privilegiando siempre al segmento de estudiantes de colegios particulares pagados. En estos establecimientos, al disponer de mayores recursos, se habilita también una mayor oferta de actividades escolares de libre elección, impactando por consecuencia en mejores tasas de participación escolar.

En la medida que se observa un comportamiento diferencial con base en la dependencia escolar, se instala una pérdida de legitimidad que pone en entredicho el sentido de una real democracia, donde cada ciudadano se haga partícipe de la sociedad que le cobija y sea portador de una membresía que le dote derechos y deberes.

Para inhibir los efectos de la segmentación y conseguir mejores resultados en toda la población estudiantil, se hace necesario intervenir desde un enfoque promocional en la escuela, requiriendo para este objetivo el compromiso de toda la comunidad educativa. El trabajo docente en aula, los talleres y programas que realizan los asistentes de la educación, la articulación con las familias y el trabajo en red con instituciones externas pueden constituir, dada la naturaleza no estrictamente académica de la participación y la formación ciudadana, estrategias potentes para reducir su estratificación.

Por otra parte, la política educativa juega un rol importante en la reducción de la violencia estructural. Las iniciativas y reformas que persiguen una mayor mixtura social en el aula, mediante estrategias de inclusión podrían no solo mejorar la cohesión, sino que colateralmente impactar de forma positiva en las competencias y habilidades civiles y cívicas del alumnado.

Una sociedad que pretende ser democrática no puede prescindir del rol formador de la escuela en estos aspectos. Esta debe educar a todas y todos para que sean 
capaces de participar de la idea de país que se anhela, definiciones colectivas que operan desde la formación de la inteligencia del saber y de las virtudes ciudadanas del ser, contemplando la necesidad de respeto y la tolerancia que aumenten la cohesión social y mejoren los espacios de convivencia futuro entre grupos sociales distintos, pero que se perciben como iguales y legítimos. En un entorno caracterizado por la individuación y relegamiento de lo colectivo, el accionar de la escuela puede activar la importancia de los valores sociales construyendo un espacio para la expresión democrática a través de la formación ciudadana como acción pedagógica intencionada.

Esta inversión de tiempo pedagógico en la formación para la vida democrática tiene un valor no solo simbólico, sino que es socialmente redituable y posiciona a la escuela como una institución especialista en formación de personas para vivir en sociedad. Si bien el debate actual sobre la calidad de la educación tiende a ignorar la importancia de estos indicadores no cognitivos del currículum, resulta cada vez más evidente que el desarrollo de habilidades sociales tiene incidencia en el tipo de sociedad que se construye, así como en el clima de convivencia imperante en la escuela. Densificación de valores sociales que puede mitigar la segmentación estructural del espacio educativo, aumentar la cohesión social y mejorar el clima de convivencia escolar, haciendo de la escuela un entorno de integración donde el estudiante pueda sentirse emocionalmente a salvo.

\section{REFERENCIAS}

Agudelo, A., Murillo, L., Echeverry, L., y Patiño, J. (2013). Participación ciudadana y prácticas políticas de jóvenes en la cotidianidad. Revista Latinoamericana de Ciencias Sociales, Niñez y Juventud, 11(2), 587-602.

Antelo, E. (2007). Variaciones sobre el espacio escolar. En R. Baquero, G. Diker y G. Frigerio (Eds.), Las formas de lo escolar (pp. 59-78). Buenos Aires, Argentina: Editorial del Estante.

Bascopé, M., Cox, C., y Lira, R. (2015). Tipos de ciudadanos en los currículos del autoritarismo y la democracia. En C. Cox y J. Castillo (Eds.), Aprendizaje de la ciudadanía. Contextos, experiencias y resultados (pp. 245-281). Santiago de Chile, Chile: Ediciones UC.

Bellei, C., Canales, M., y Orellana, V. (2015). El soporte cultural para el mercado educacional. Disposiciones culturales y prácticas de las familias chilenas referidas a la elección de escuelas. Santiago de Chile, Chile: FONDECYT 1130430.

Cohen, E., y Franco, R. (1992). Evaluación de proyectos sociales. México D. F., México: Siglo XXI.

Contreras, G., y Morales, M. (2014). Jóvenes y participación electoral en Chile 1989-2013. Analizando el efecto del voto voluntario. Revista Latinoamericana de Ciencias Sociales, Niñez y Juventud, 12(2), 597-615. 
Corvalan, A., y Cox, P. (2015). Participación y desigualdad electoral en Chile. En C. Cox y J. Castillo (Eds.), Aprendizaje de la ciudadanía. Contextos, experiencias y resultados (pp. 175-204). Santiago de Chile, Chile: Ediciones UC.

Cox, C., y Castillo, J. (2015). Aprendizaje de la ciudadanía: Contextos, experiencias y resultados. Santiago de Chile, Chile: Ediciones UC.

Cox, C., Jaramillo, R., y Reimers, F. (2005). Educar para la ciudadanía y la democracia en las Américas: Una agenda para la acción. Presentado en el seminario previo a la IV Reunión de Ministros de Educación, Banco Interamericano de Desarrollo, Trinidad y Tobago.

Díaz, G. (2014). Educación para la ciudadanía y participación electoral en un contexto de voto voluntario. Revista de Ciencia Política, 52(1), 61-91.

Gasparini, L., y Molina, E. (2006). Income Distribution, Institutions and Conflicts: An Exploratory Analysis for Latin América and the Caribbean. Recuperado de http://sedici.unlp.edu.ar/bitstream/handle/10915/3600/Documento_completo__.pdf?sequence=1

Hernández, M., y Raczynski, D. (2015). Elección de escuela en Chile: De las dinámicas de distinción y exclusión a la segregación socioeconómica del sistema escolar. Estudios Pedagógicos, 41(2), 127-141. https://doi.org/10.4067/S0718-07052015000200008

Lafferte, C., y Silva Gallinato, S. (Eds.). (2014). La voz del movimiento estudiantil 2011. Educación pública, gratuita y de calidad. Santiago de Chile, Chile: Fondo de las Naciones Unidas para la Infancia.

Mardones, R. (2015). El paradigma de la educación ciudadana en Chile: una política pública inconclusa. En C. Cox y J. Castillo (Eds.), Aprendizaje de la ciudadanía. Contextos, experiencias y resultados (pp. 145-173). Santiago de Chile, Chile: Ediciones UC.

Marshall, T., y Bottomore, T. (1998). Ciudadanía y clase social. Madrid, España: Editorial Alianza.

Mieles, M. D., y García, M. C. (2010). Apuntes sobre socialización infantil y construcción de identidad en ambientes multiculturales. Revista Latinoamericana de Ciencias Sociales, Niñez y Juventud, 8(2), 809-819.

Muñoz, G. (2011a). La democracia y la participación en la escuela: ¿cuánto se ha avanzado desde las normativas para promover la participación escolar?. Revista de Estudios y Experiencias en Educación, 10(19), 107-129.

Muñoz, G. (2011b). Representación simbólica de los consejos escolares como estrategia para democratizar la cultura escolar: una lectura interpretativa desde la voz de los sujetos sociales. Estudios Pedagógicos, 37(1), 35-52. https://doi.org/10.4067/S0718-07052011000100002

Ortiz, I. (2015). Escuelas Inclusivas en el contexto de segregación social del sistema escolar chileno. Calidad en la Educación, (42), 93-122. https://doi.org/10.4067/S0718-45652015000100004

Peña, C. (2007). Educación y ciudadanía: los problemas subyacentes. Pensamiento Educativo, 40(1), 31-43.

Redon, S. (2010). La escuela como espacio de ciudadanía. Estudios Pedagógicos, 36(2), 213-239. https://doi.org/10.4067/S0718-07052010000200013 
Rivera, R., y Santos, D. (2015). Participación de los niños y educación cívica: bases para un modelo social inclusivo. Revista Iberoamericana de Educación, 67, 37-52.

Rosas, R., y Santa Cruz, C. (2013). Dime en qué colegio estudiaste y te diré qué Cl tienes: Radiografía al desigual acceso al capital cognitivo en Chile. Santiago de Chile, Chile: Ediciones UC.

Sarramona, J., y Rodríguez, T. (2010). Participación y calidad de la educación. Aula Abierta, 38(1), 3-14.

Schulz, W., Fraillon, J., Ainley, J., Losito, B., y Kerr, D. (2009). Estudio Internacional sobre Educación Cívica y Ciudadana. Madrid, España: Ministerio de Educación de España.

Vergara, E., Montaño, N., Becerra, R., León-Enríquez, O., y Arboleda, C. (2011). Prácticas para la formación democrática en la escuela: ¿Utopía o realidad?. Revista Latinoamericana de Ciencias Sociales, Niñez y Juventud, 9(1), 227-253.

Vargas, S., Oyanedel, J., y Torres, J. (2015). Socialización e interés en la política en Chile. Revista Latinoamericana de Ciencias Sociales, Niñez y Juventud, 13(2), 781-794. 